\title{
Spectral properties of discrete alloy-type models
}

\author{
Martin Tautenhahn and Ivan Veselić \\ Emmy-Noether-Projekt Schrödingeroperatoren, Fakultät für Mathematik, \\ Technische Universität Chemnitz, 09127 Chemnitz, Germany \\ www.tu-chemnitz.de/mathematik/enp/
}

\begin{abstract}
We discuss recent results on spectral properties of discrete alloy-type random Schrödinger operators. They concern Wegner estimates and bounds on the fractional moments of the Green's function.

Keywords: random Schrödinger operators; discrete alloy-type model; single-site potential.
\end{abstract}

\section{Introduction and model}

A discrete alloy-type model is a family of operators $H_{\omega}=H_{0}+V_{\omega}$ on $\ell^{2}\left(\mathbb{Z}^{d}\right)$ where $H_{0}$ is the negative discrete Laplacian given by $\left(H_{0} \psi\right)(x)=-\sum_{|e|=1} \psi(x+e)$, and $V_{\omega}$ the multiplication operator by the function

$$
V_{\omega}(x)=\sum_{k \in \mathbb{Z}^{d}} \omega_{k} u(x-k) .
$$

Here $\omega_{k}, k \in \mathbb{Z}^{d}$, is an i. i.d. sequence of random variables and $u: \mathbb{Z}^{d} \rightarrow \mathbb{R}$ a socalled single-site potential. The minimal conditions which are assumed throughout this note are that the distribution of $\omega_{0}$ has a density $\rho \in L^{\infty}(\mathbb{R})$ and that $u$ belongs to $\ell^{1}\left(\mathbb{Z}^{d}\right)$. There are several well-studied relatives of the discrete alloy-type model:

(i) The continuum alloy-type model $-\Delta+V_{\omega}$ on $L^{2}\left(\mathbb{R}^{d}\right)$ where $V_{\omega}$ has the same form as in (11).

(ii) The Anderson model on $\ell^{2}\left(\mathbb{Z}^{d}\right)$ : this is the special case of the discrete alloy-type model where $u=\delta_{0}$.

(iii) The correlated Anderson model, which corresponds to $u=\delta_{0}$ and the case where the random coupling constants $\omega_{k}$ are no longer assumed independent.

Obviously, the discrete alloy-type model is a special case of case (iii). However, previous results on (iii)-type models require certain regularity conditions which are typically not satisfied for alloy-type potentials, cf. Section 3

The main challenge of models of the type (11) is that $u$ may change its sign which leads to negative correlations of the potential. This results in a non-monotone dependence on the random variables of certain spectral quantities. For this reason many established tools for the spectral analysis of random Schrödinger operators are not directly applicable in this model. 
For literature concerning the discussion in this section we refer to the references of [1, 2].

\section{Results}

Our results concern averages of resolvents and projectors. The presented results are closely related to dynamical and spectral localisation. We use the symbol $\mathbb{E}$ to denote the average over the collection of random variables $\omega_{k}, k \in \mathbb{Z}^{d}$. For $\Lambda \subset \mathbb{Z}^{d}$ we denote by $H_{\omega, \Lambda}: \ell^{2}(\Lambda) \rightarrow \ell^{2}(\Lambda)$ the natural restriction of $H_{\omega}$ to the set $\Lambda$.

\subsection{Wegner estimate}

We present here a selection of the results proven in [2]. For $L \in \mathbb{N}$ we denote the set $[0, L]^{d} \cap \mathbb{Z}^{d}$ by $\Lambda_{L}$. The number of points in the support of $u$ is denoted by rank $u$.

Theorem 2.1. Assume that the single-site potential $u$ has support in $\Lambda_{n}$ and $\rho$ is of bounded variation. Then there exists a constant $c_{u}$ depending only on $u$ such that for any $L \in \mathbb{N}, E \in \mathbb{R}$ and $\epsilon>0$ we have

$$
\mathbb{E}\left\{\operatorname{Tr}\left[\chi_{[E-\epsilon, E-\epsilon]}\left(H_{\omega, \Lambda_{L}}\right)\right]\right\} \leq c_{u}\|\rho\|_{\operatorname{Var}} \operatorname{rank} u \epsilon(L+n)^{d \cdot(n+1)} .
$$

Here $\|\rho\|_{\text {Var }}$ denotes the total variation of $\rho$. By the assumption on the support of the single-site potential, $\operatorname{rank} u \leq n^{d}$. Our bound is linear in the energy-interval length and polynomial in the volume of the cube $\Lambda_{L}$. This implies that the Wegner bound can be used for a localisation proof via multiscale analysis, as soon as an appropriate initial length scale estimate is at disposal.

Theorem 2.2. Assume $\bar{u}:=\sum_{k \in \mathbb{Z}^{d}} u(k) \neq 0$ and that $\rho$ has compact support. Let $m \in \mathbb{N}$ be such that $\sum_{\|k\| \geq m}|u(k)| \leq|\bar{u} / 2|$. Then we have for any $L \in \mathbb{N}, E \in \mathbb{R}$ and $\epsilon>0$

$$
\mathbb{E}\left\{\operatorname{Tr}\left[\chi_{[E-\epsilon, E-\epsilon]}\left(H_{\omega, \Lambda_{L}}\right)\right]\right\} \leq \frac{8}{\bar{u}}\|\rho\|_{\text {Var }} \min \left(L^{d}, \operatorname{rank} u\right) \epsilon(L+m)^{d} .
$$

In the case that the support of $u$ is compact we obtain a bound which is linear in the volume of the box and thus yields the Lipschitz continuity of the integrated density of states.

\subsection{Boundedness of fractional moments of Green's function}

In this subsection we assume that the function $\hat{u}:[0,2 \pi)^{d} \rightarrow \mathbb{C}$, defined by

$$
\hat{u}(\theta)=\sum_{k \in \mathbb{Z}^{d}} u(k) \mathrm{e}^{\mathrm{i} k \cdot \theta}
$$

does not vanish, supp $u$ is compact, and $\rho \in W^{1,1}(\mathbb{R})$. Let $\Lambda \subset \mathbb{Z}^{d}$ be finite. For $x, y \in \Lambda$ and $z \in \mathbb{C} \backslash \mathbb{R}$ we set $G_{\omega, \Lambda}(z ; x, y)=\left\langle\delta_{x},\left(H_{\omega, \Lambda}-z\right)^{-1} \delta_{y}\right\rangle$. 
Theorem 2.3. Let $s \in(0,1)$ and $\Lambda \subset \mathbb{Z}^{d}$ be finite. Then there exists a constant $C_{u}$ depending only on $u$, such that for all $z \in \mathbb{C} \backslash \mathbb{R}$ and all $x, y \in \Lambda$

$$
\mathbb{E}\left\{\left|G_{\omega, \Lambda}(z ; x, y)\right|^{s}\right\} \leq\left(C_{u}\left\|\rho^{\prime}\right\|_{L^{1}}\right) \frac{s 2^{1+s} s^{-s}}{1-s} .
$$

Theorem 2.3 is proven in Section 4 . The proof is a combination of ideas from 3 . and 4 . The proof also gives rise to a quantitative estimate on the constant $C_{u}$. To our knowledge, Theorem 2.3 does not immediately imply exponential decay of fractional moments of the Green's function by standard methods.

\subsection{Exponential decay of fractional moments of Green's function}

In this subsection we assume $d=1$ and $\operatorname{supp} u$ compact. Denote the diameter of the support by $n-1 \in \mathbb{N}_{0}$. We define the following conditions which may or may not hold:

(A) $\operatorname{supp} u=\{0,1, \ldots, n-1\}$,

(B) $\operatorname{supp} \rho$ compact,

(C) $\rho \in W^{1,1}(\mathbb{R})$.

For $x, y \in \mathbb{Z}^{d}$ and $z \in \mathbb{C} \backslash \mathbb{R}$ we denote the Green's function by $G_{\omega}(z ; x, y)=$ $\left\langle\delta_{x},\left(H_{\omega}-z\right)^{-1} \delta_{y}\right\rangle$ and set

$$
C_{u, \rho}=\left|\prod_{k \in \Theta} u(k)\right|^{-s / n}\|\rho\|_{\infty}^{s} \frac{2^{s} s^{-s}}{1-s} .
$$

Theorem 2.4. Let $s \in(0,1)$ and assume that $(A)$ holds. Then there exists a constant $C$, depending on $\|\rho\|_{\infty}, s$ and $u$, such that for all $x, y \in \mathbb{Z}$ with $|x-y| \geq n$ and all $z \in \mathbb{C} \backslash \mathbb{R}$ we have

$$
\mathbb{E}\left\{\left|G_{\omega}(z ; x, y)\right|^{s / n}\right\} \leq C \exp \left\{-m\left\lfloor\frac{|x-y|}{n}\right\rfloor\right\},
$$

where $m=-\ln C_{u, \rho}$ and $\lfloor\cdot\rfloor$ is defined by $\lfloor t\rfloor=\max \{k \in \mathbb{Z}: k \leq t\}$.

If $C_{u, \rho}<1$, or equivalently $m>0$, Ineq. (2) describes exponential decay.

Theorem 2.5. Let $s \in(0,1 / 2)$ and assume that either $(B)$ holds with $\|\rho\|_{\infty}$ sufficiently small, or $(C)$ holds with $\left\|\rho^{\prime}\right\|_{L^{1}}$ sufficiently small. Then there exist constants $m, C \in(0, \infty)$, such that Eq. (2) holds for all $x, y \in \mathbb{Z}$ with $|x-y| \geq 4 n$ and all $z \in \mathbb{C} \backslash \mathbb{R}$.

Theorem 2.4 and 2.5 are proven in a joint paper [1] with A. Elgart. The estimates of Theorem 2.4 and 2.5 concern only off-diagonal elements. If we assume (B) and $s \in(0,1 / 4 n)$, then $\mathbb{E}\left\{\left|G_{\omega}(z ; x, y)\right|^{s}\right\}$ is uniformly bounded for $x, y \in \mathbb{Z}$ and $z \in$ $\mathbb{C} \backslash \mathbb{R}$, see [1, Appendix]. In spite these estimates on the Green's function, neither dynamical nor spectral localisation follow immediately using the existent methods. 
Currently, the analogues of Theorem 2.4 and2.5 in higher dimension are an open question. In a forthcoming paper with A. Elgart we intend to extend Theorem 2.5 to operators on a one-dimensional strip, i.e. $\Lambda=\Gamma \times \mathbb{Z}$ with $\Gamma \subset \mathbb{Z}^{d}$ finite.

\section{Example}

We show that discrete alloy-type models do not satisfy in general the regularity conditions required in [5, 6] for correlated Anderson models. We consider $d=1$. Let the density function $\rho$ be of bounded support and let $u(0)=1, u(-1)=a \neq 0$ and $u(k)=0$ for $k \in \mathbb{Z} \backslash\{-1,0\}$. For simplicity we require that the infimum of the support of $\rho$ is zero. Let $B_{\epsilon}$ and $A_{\epsilon}$ be the events $B_{\epsilon}=\left\{\omega \in \Omega: V_{\omega}(-1), V_{\omega}(1) \in\right.$ $[0, \epsilon]\}$ and $A_{\epsilon}=\left\{\omega \in \Omega: V_{\omega}(0) \in\left[0, \epsilon\left(a+\frac{1}{a}\right)\right]\right\}$ with $\epsilon>0$. Then, if $a>0$ one calculates $B_{\epsilon} \subset A_{\epsilon}$ for all $\epsilon>0$ and consequently we have

$$
\mathbb{P}\left\{A_{\epsilon} \mid B_{\epsilon}\right\}=\frac{\mathbb{P}\left\{A_{\epsilon} \cap B_{\epsilon}\right\}}{\mathbb{P}\left\{B_{\epsilon}\right\}}=1 \quad \forall \epsilon>0 .
$$

This shows that the regularity assumptions required in [5, 6] are violated. In the case $a<0$ one can proceed analogously, but with a different choice of the sets $A_{\epsilon}$ and $B_{\epsilon}$.

\section{Proof of Theorem 2.3}

Let $L \in \mathbb{N}$ be such that the cube $\Lambda^{+}=[-L, L]^{d} \cap \mathbb{Z}^{d}$ contains $\bigcup_{x \in \Lambda}\left\{k \in \mathbb{Z}^{d}: u(x-\right.$ $k) \neq 0\}$, which is the set of all lattice sites whose coupling constant influence the potential in $\Lambda$. Let $A: \ell^{1}\left(\mathbb{Z}^{d}\right) \rightarrow \ell^{1}\left(\mathbb{Z}^{d}\right)$ be the linear operator whose coefficients in the canonical orthonormal basis are $A(j, k)=u(j-k)$ for $j, k \in \mathbb{Z}^{d}$. Since $u$ has compact support, the operator $A$ is bounded. Since $\hat{u}$ does not vanish, $C_{u}=$ $\left\|A^{-1}\right\|_{1}<\infty$, see [4] for details. Moreover, there exists an invertible matrix $A_{\Lambda^{+}}$: $\ell^{1}\left(\Lambda^{+}\right) \rightarrow \ell^{1}\left(\Lambda^{+}\right)$satisfying

$$
A_{\Lambda^{+}}(j, k)=u(j-k) \quad \text { for all } j \in \Lambda \text { and } k \in \Lambda^{+}
$$

and $\left\|A_{\Lambda^{+}}^{-1}\right\| \leq C_{u}$, see [4, Proposition 5]. We set $B_{\Lambda^{+}}=A_{\Lambda^{+}}^{-1}$. From [5] and [7], respectively, we infer that for $x, y \in \Lambda$ with $x \neq y$

$$
\left|G_{\omega, \Lambda}(z ; x, x)\right|=\frac{1}{\left|V_{\omega}(x)-\alpha\right|} \text { and }\left|G_{\omega, \Lambda}(z ; x, y)\right| \leq \frac{2}{\left|V_{\omega}(x)-\beta\right|}+\frac{2}{\left|V_{\omega}(y)-\gamma\right|} .
$$

Here $\alpha, \beta \in \mathbb{C}$ are functions of $V_{\omega}(k), k \in \Lambda \backslash\{x\}$, and $\gamma \in \mathbb{C}$ is a function of $V_{\omega}(k)$, $k \in \Lambda \backslash\{y\}$. Set $\omega_{\Lambda^{+}}=\left(\omega_{k}\right)_{k \in \Lambda^{+}}, k\left(\omega_{\Lambda^{+}}\right)=\prod_{k \in \Lambda^{+}} \rho\left(\omega_{k}\right), \mathrm{d} \omega_{\Lambda^{+}}=\prod_{k \in \Lambda^{+}} \mathrm{d} \omega_{k}$ and $\Omega_{\Lambda^{+}}=\times_{k \in \Lambda^{+}} \mathbb{R}$. Using the substitution $\zeta_{\Lambda^{+}}=\left(\zeta_{k}\right)_{k \in \Lambda^{+}}=A_{\Lambda^{+}} \omega_{\Lambda^{+}}$and Eq. (3) we obtain

$$
S:=\int_{\Omega_{\Lambda^{+}}} \frac{1}{\left|V_{\omega}(x)-\alpha\right|^{s}} k\left(\omega_{\Lambda^{+}}\right) \mathrm{d} \omega_{\Lambda^{+}}=\int_{\Omega_{\Lambda^{+}}} \frac{1}{\left|\zeta_{x}-\alpha\right|^{s}} k\left(B_{\Lambda^{+}} \zeta_{\Lambda^{+}}\right)\left|\operatorname{det} B_{\Lambda^{+}}\right| \mathrm{d} \zeta_{\Lambda^{+}},
$$


where $\mathrm{d} \zeta_{\Lambda^{+}}=\prod_{k \in \Lambda^{+}} \mathrm{d} \zeta_{k}$. Since by construction $\zeta_{k}=V_{\omega}(k)$ for all $k \in \Lambda, \alpha$ is now a function of $\zeta_{k}, k \in \Lambda \backslash\{x\}$. For non-negative functions $g: \mathbb{R} \rightarrow \mathbb{R}$ with $g \in W^{1,1}(\mathbb{R})$ and $\delta \in \mathbb{C}$ one has for all $\lambda>0$ the estimate

$$
\int_{\mathbb{R}} \frac{g(\xi)}{|\xi-\delta|^{s}} \mathrm{~d} \xi \leq \lambda^{-s}\|g\|_{L^{1}}+\|g\|_{\infty} \frac{2 \lambda^{1-s}}{1-s} \leq \lambda^{-s}\|g\|_{L^{1}}+\left\|g^{\prime}\right\|_{L^{1}} \frac{\lambda^{1-s}}{1-s},
$$

where the first inequality is due to [7] and the second is the fundamental theorem of calculus. Set $\Omega_{\Lambda^{+}}^{x}=\times_{k \in \Lambda^{+} \backslash\{x\}} \mathbb{R}$. Using Fubini's theorem and Ineq. (5) we obtain

$$
\begin{aligned}
S & \leq \int_{\Omega_{\Lambda^{x}}}\left(\lambda^{-s} \int_{\mathbb{R}} k\left(B_{\Lambda^{+}} \zeta_{\Lambda^{+}}\right) \mathrm{d} \zeta_{x}+\frac{\lambda^{1-s}}{1-s} \int_{\mathbb{R}}\left|\frac{\partial}{\partial \zeta_{x}} k\left(B_{\Lambda^{+}} \zeta_{\Lambda^{+}}\right)\right| \mathrm{d} \zeta_{x}\right)\left|\operatorname{det} B_{\Lambda^{+}}\right| \prod_{k \in \Lambda^{+} \backslash\{x\}} \mathrm{d} \zeta_{k} \\
& =\lambda^{-s}+\frac{\lambda^{1-s}}{1-s} \int_{\Omega_{\Lambda^{+}}}\left|\frac{\partial}{\partial \zeta_{x}} k\left(B_{\Lambda^{+}} \zeta_{\Lambda^{+}}\right)\right| \operatorname{det} B_{\Lambda^{+}} \mid \mathrm{d} \zeta_{\Lambda^{+}} .
\end{aligned}
$$

We calculate the partial derivative by the product rule, substitute back into original coordinates and obtain

$$
\begin{aligned}
S & \leq \lambda^{-s}+\frac{\lambda^{1-s}}{1-s} \int\left|\sum_{\Omega_{\Lambda^{+}} \in \Lambda^{+}} \rho^{\prime}\left(\left(B_{\Lambda^{+}} \zeta_{\Lambda^{+}}\right)_{j}\right) B_{\Lambda^{+}}(j, x) \prod_{\substack{k \in \Lambda^{+} \\
k \neq j}} \rho\left(\left(B_{\Lambda^{+}} \zeta_{\Lambda^{+}}\right)_{k}\right)\right|\left|\operatorname{det} B_{\Lambda^{+}}\right| \mathrm{d} \zeta_{\Lambda^{+}} \\
& \leq \lambda^{-s}+\frac{\lambda^{1-s}}{1-s} \sum_{j \in \Lambda^{+}}\left|B_{\Lambda^{+}}(j, x)\right| \int_{\Omega_{\Lambda^{+}}}\left|\rho^{\prime}\left(\omega_{j}\right)\right| \prod_{\substack{k \in \Lambda^{+} \\
k \neq j}} \rho\left(\omega_{k}\right) \mathrm{d} \omega_{\Lambda^{+}} \\
& =\lambda^{-s}+\frac{\lambda^{1-s}}{1-s}\left\|B_{\Lambda^{+}}\right\|\left\|_{1}\right\| \rho^{\prime}\left\|_{L^{1}} \leq \lambda^{-s}+\frac{\lambda^{1-s}}{1-s} C_{u}\right\| \rho^{\prime} \|_{L^{1}} .
\end{aligned}
$$

If we choose $\lambda=s /\left(C_{u}\left\|\rho^{\prime}\right\|_{L^{1}}\right)$ we obtain $S \leq C_{u}^{s}\left\|\rho^{\prime}\right\|_{L^{1}}^{s} s^{-s} /(1-s)$. Thus we have shown $\mathbb{E}\left\{\left|G_{\omega, \Lambda}(z ; x, x)\right|^{s}\right\} \leq C_{u}^{s}\left\|\rho^{\prime}\right\|_{L^{1}}^{s} \frac{s^{-s}}{1-s}$. Analogously we obtain for $x \neq y$ the estimate $\mathbb{E}\left\{\left|G_{\omega, \Lambda}(z ; x, y)\right|^{s}\right\} \leq 2^{s+1} C_{u}^{s}\left\|\rho^{\prime}\right\|_{L^{1}}^{s} \frac{s^{-s}}{1-s}$. The additional factor $2^{s+1}$ arises in the case $x \neq y$, since in Eq. (4) there are two summands of the type $S$, and each summand has an additional factor 2 in its numerator.

\section{References}

[1] A. Elgart, M. Tautenhahn and I. Veselić, Exponential decay of Green's function for Anderson models on $\mathbb{Z}$ with single-site potentials of finite support, Preprint, arXiv:0903.0492 [math-ph], (2009).

[2] I. Veselić, Wegner estimates for discrete alloy-type models, Preprint, mp_arc no. 09100, (2009).

[3] M. Tautenhahn, Lokalisierung für korrelierte Anderson Modelle, Diplomarbeit, Techn. Univ. Chemnitz (2007).

[4] I. Veselić, Wegner estimates for sign-changing single site potentials, Preprint, arXiv:0806.0482 [math.SP], (2008).

[5] M. Aizenman and S. Molchanov, Commun. Math. Phys. 157, 245 (1993).

[6] M. Aizenman, J. H. Schenker, R. M. Friedrich and D. Hundertmark, Commun. Math. Phys. 224, 219 (2001).

[7] G. M. Graf, J. Stat. Phys. 75, 337 (1994). 